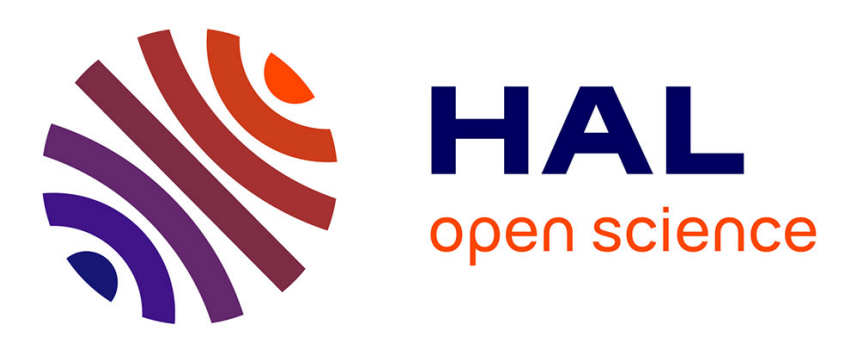

\title{
Impact of distributed energy resources and electric vehicle smart charging on low voltage grid stability
}

Felipe Gonzalez, Simon Meunier, Christina Protopapadaki, Yannick Perez, Dirk Saelens, Marc Petit

\section{- To cite this version:}

Felipe Gonzalez, Simon Meunier, Christina Protopapadaki, Yannick Perez, Dirk Saelens, et al.. Impact of distributed energy resources and electric vehicle smart charging on low voltage grid stability. CIRED 2021, Sep 2021, Online, France. hal-03420069

\section{HAL Id: hal-03420069 https://hal.science/hal-03420069}

Submitted on 8 Nov 2021

HAL is a multi-disciplinary open access archive for the deposit and dissemination of scientific research documents, whether they are published or not. The documents may come from teaching and research institutions in France or abroad, or from public or private research centers.
L'archive ouverte pluridisciplinaire HAL, est destinée au dépôt et à la diffusion de documents scientifiques de niveau recherche, publiés ou non, émanant des établissements d'enseignement et de recherche français ou étrangers, des laboratoires publics ou privés. 


\title{
Impact of distributed energy resources and electric vehicle smart charging on low voltage grid stability Felipe GONZALEZ VENEGAS ${ }^{1,2,3 *}$, Simon MEUNIER ${ }^{1,2,4}$, Christina PROTOPAPADAKI I, , Yannick PEREZ ${ }^{6}$, Dirk SAELENS ${ }^{4,5}$, Marc PETIT ${ }^{1,2}$
}

\author{
${ }^{1}$ Université Paris-Saclay, CentraleSupélec, CNRS, GeePs, Gif-sur-Yvette 91192, France \\ ${ }^{2}$ Sorbonne Université, CNRS, GeePs, Paris 75252, France \\ ${ }^{3}$ Innovation \& Advanced Technologies Research Division, Stellantis, 78955, Carrières-sous-Poissy, France \\ ${ }^{4}$ KU Leuven, Department of Civil Engineering, Building Physics Section, Heverlee 3001, Belgium \\ ${ }^{5}$ EnergyVille, Genk 3600, Belgium \\ ${ }^{6}$ Laboratoire de Génie Industriel, CentraleSupélec, Gif-sur-Yvette 91192, France \\ *felipe.gonzalezvenegas@centralesupelec.fr
}

\begin{abstract}
Keywords: ELECTRIC VEHICLES, LOW VOLTAGE DISTRIBUTION NETWORKS, DISTRIBUTED ENERGY RESOURCES, HEAT PUMPS, PV SYSTEMS
\end{abstract}

\begin{abstract}
Environmental policies are pushing cross-sector electrification, including the transport and heating sectors. However, the large integration of distributed energy resources, such as electric vehicles (EV), heat pumps (HP), and photovoltaic (PV) systems, has a strong influence on the stability of low voltage (LV) distribution grids and can lead to high reinforcement costs. This work develops a methodology to quantify the impacts of a joint integration of EVs, PV, and HPs into LV distribution grids and the ability of a decentralized EV smart charging strategy to reduce the constraints. A central occupancy model generates demand and generation profiles for the studied technologies, which are then used to carry out Monte Carlo simulations for unbalanced power flow analysis of LV grids. A case study of a typical Belgian rural grid shows that the main impacts arise from HP integration, creating voltage deviations and transformer overloading. EV integration impacts are less significant, creating grid issues only at high-penetration levels and to a lesser extent than HPs. The proposed smart charging strategy allows reducing the impacts of EV integration; however, it does not allow increased penetration of the other technologies. Other smart charging and Vehicle-to-Grid (V2G) strategies should be analyzed to further increase DER penetration.
\end{abstract}

\section{Introduction}

Environmental policies are pushing cross-sector electrification, including the transport and heating sectors. The integration of distributed energy resources (DER) such as electric vehicles (EV) and heat pumps (HP) coupled with low carbon generation such as photovoltaic (PV) panels can greatly reduce $\mathrm{CO}_{2}$ emissions needed to limit the impact of climate change. However, the large integration of DERs can have a strong impact on the stability of low-voltage (LV) distribution grids, by creating voltage or thermal issues on grid assets, and can lead to significant reinforcement costs for distribution system operators (DSO).

The present work proposes a methodology to quantify the impacts of the joint integration of high shares of EVs, PV systems, and HPs into LV grids, and the ability of EV smart charging to reduce the grid constraints.

The main contribution of this methodology is the analysis of the joint integration of these three DERs through advanced building and occupancy models. Most studies and methods focus on only one or two of these three technologies, such as [1][2] for PV and HP, or use independently generated load curves for each one of them, such as in [3] [4] [5]. In this work, through a central dwelling model, all building loads and sources (baseload, $\mathrm{EV}, \mathrm{HP}$ and $\mathrm{PV}$ ) derive from a single common origin, allowing for coherent profiles for each technology in each household. Furthermore, we are able to compare different EV charging strategies that take into account the other DERs. Finally, detailed power-flow simulations allow identifying the impact of DER integration on grid stability. This methodology is then applied to a typical Belgian rural LV network to evaluate the impact of EV, HP and PV integration and EV smart charging.

In Section 2, we present the methodology developed to evaluate the impact of high shares of DERs into LV grids. Then, we present the case study in Section 3 and the simulations' results in Section 4. This work ends with the conclusions and an outlook for future work in Section 5 .

\section{Method and modeling}

As shown in Figure 1, the methodology developed is based on two sequential models: a dwelling and a grid model, described in the following subsections. 


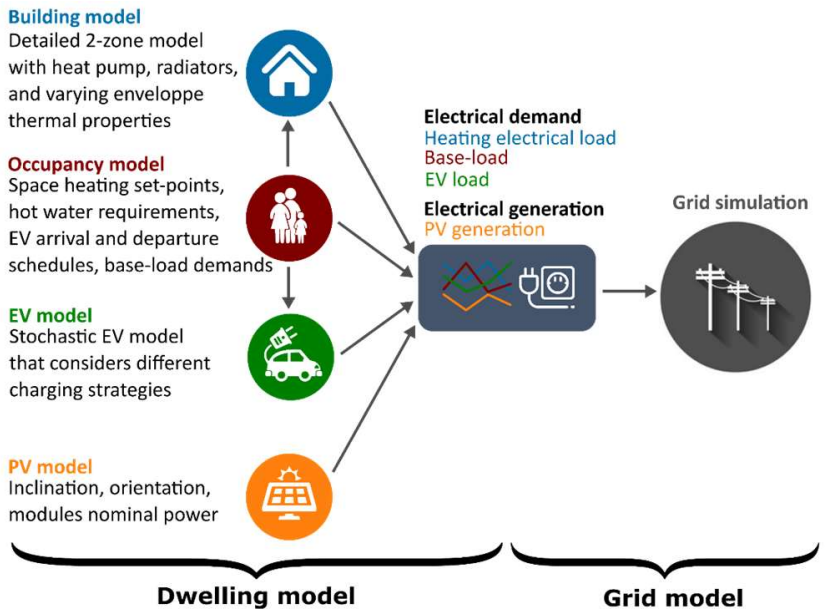

Figure 1: Methodology overview

\subsection{Dwelling model and EV charging strategies}

The dwelling model simulates the electrical demand and generation profiles of each dwelling on the grid. It is composed of four main components namely the occupancy, building, EV and PV models.

The occupancy model defines the set of occupant profiles of each dwelling. The occupant profiles capture a wide diversity of habits, and provides the heating requirements of the inhabitants and the EV arrival and departure schedules. The occupancy model also simulates the base load, corresponding to the electricity use for lighting, domestic appliances and electronics.

The building model then generates the heating electrical demand for the given building properties, occupants, HP characteristics, and weather conditions. This profile includes HP operation, domestic water heating and back-up heaters. Furthermore, PV generation is simulated for the same weather and the PV installation size and orientation.

The EV model generates the EV load profiles for each dwelling. Arrival and departure times are derived from occupancy schedules. Travelled distances for each trip are computed considering different occupant characteristics and trip motives, including work, shopping and leisure among others, following the methodology from [6]. We considered as well non-systematic plug-in behavior, which emulates behavior observed in real life conditions where users do not plug in their EV every day. A stochastic plug in decision model, described in [7], defines whether the EV is plugged in for each possible charging session.

Two EV charging strategies are compared: uncoordinated charging and decentralized smart charging. The uncoordinated charging starts as soon as the vehicle is plugged in, and charges at maximum power until the EV battery is fully charged. In the decentralized smart charging strategy, the EV is charged to minimize the electricity costs of end-users while also respecting the maximum contracted power of the whole building. This type of smart charging can be achieved by a home energy management system. We considered an asynchronous time-of-use (peak/off-peak) tariff for end-users, where each household's off-peak period starts at a slightly different time to avoid synchronization of EV charging at the transformer level.

\subsection{Grid model}

The grid model analyzes the grid's stability at various DER penetration levels, as in [8]. Monte Carlo simulations for unbalanced three-phase power flow analysis are carried out using Modelica. Analyzed LV grids consist of a MV/LV transformer, a detailed LV feeder, and a dummy feeder representing the loads in the rest of the grid. For each DER penetration level, several iterations (i.e., yearly power flow simulations) are evaluated, each considering different dwelling types, occupancy patterns, and DER locations in the feeder. Four indicators are considered to assess grid stability:

- Transformer overloading: the maximum transformer overloading, in percentage above the rated power.

- Overloaded feeders: the percentage of overloaded feeder sections (any single-phase or the neutral cable).

- Voltage deviations: the percentage of dwellings for which the European Standard EN 50610 criteria for voltage deviations is violated at any simulation period. Voltage at the dwelling connection should remain between 0.9 and $1.1 \mathrm{pu}$ for more than $95 \%$ of the time each week and never below 0.85 or above $1.1 \mathrm{pu}$.

- Voltage unbalances: the percentage of nodes for which the EN 50160 criteria for voltage unbalances is violated. The voltage unbalances at the node should remain below $2 \%$ for more than $95 \%$ of the time each week.

If any of these indicators is above zero (i.e. at least one asset is overloaded or voltage requirements are not satisfied in a node of the grid), the grid is considered as under constraint, and the DSO would need to consider reinforcement options.

\section{Case study description}

We applied the methodology to a typical rural LV grid in Belgium. The grid comprises 78 dwellings, with 15 of them in the detailed feeder with an average cable length between dwellings of $22 \mathrm{~m}$, as shown in Fig. 2. The building characteristics are randomly sampled from a building stock of 300 different detached houses with varying thermal properties corresponding to rural conditions in Belgium. Building, grid, and economic parameters were determined from previous studies on LV feeders and from cost data of the Belgian DSO Fluvius [8].

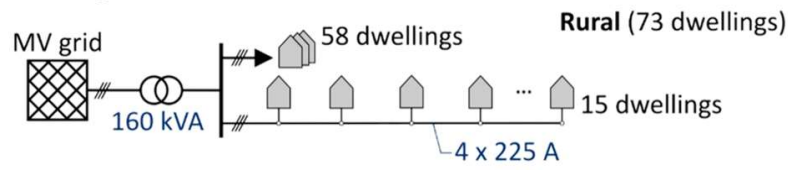

Figure 2: Typical Belgian rural LV grid considered. 
In order to capture a wide range of behaviors, the occupancy profiles consider five types of occupants: full-time employees (FTE), part-time employees (PTE), retired, unemployed, and students. There are up to five occupants in each dwelling, with at least one PTE, FTE, retired or unemployed.

DER integration scenarios: As we mostly focus on EV integration, we consider EV integration rates between 0 and $100 \%$ with a step of $20 \%$, and the combinations of $0 \%$ and $100 \%$ penetration of HP and PV. HP and PV are sized for each household according to building characteristics and occupant thermal comfort requirements, obtaining a range of HP sizes between 1.1-8.5 kWe and PV sizes between 3$5 \mathrm{kWp}[8]$.

We consider at most one EV per household that is driven only by one of its occupants. The EVs have a $50 \mathrm{kWh}$ battery pack, consistent with current trends, and are charged at home with a $7.4 \mathrm{kVA}$ charger (32 A single-phase charger, corresponding to level 2 SAE J1772 standard) [9]. The plug in decision model was calibrated to have a median plug-in frequency of 2.4 charging sessions per week, in line with the Electric Nation data, a large-scale EV trial in the UK [10]. Driven distances are derived from the occupancy profiles based on the methodology developed in [6], and calibrated with Belgian travel data. Distribution of average daily distances is shown in Figure 3, where the diversity of profiles is evidenced.

Finally, each building is considered to have a $9.2 \mathrm{kVA}$ single-phase connection, as it is the most common in Belgium. Off-peak periods are different for each dwelling, starting between midnight and 3 am, and lasting 8 hours.

Figure 4 shows the distribution of annual demand or generation by each technology. Differences in dwellings types and user preferences generate a wide range of load profiles for each dwelling and show the diversity that our model is able to capture. In terms of annual load, incorporating a HP or an EV represent roughly the base load of one extra household, which the addition of PV panels can partly compensate. However, due to the different periods of

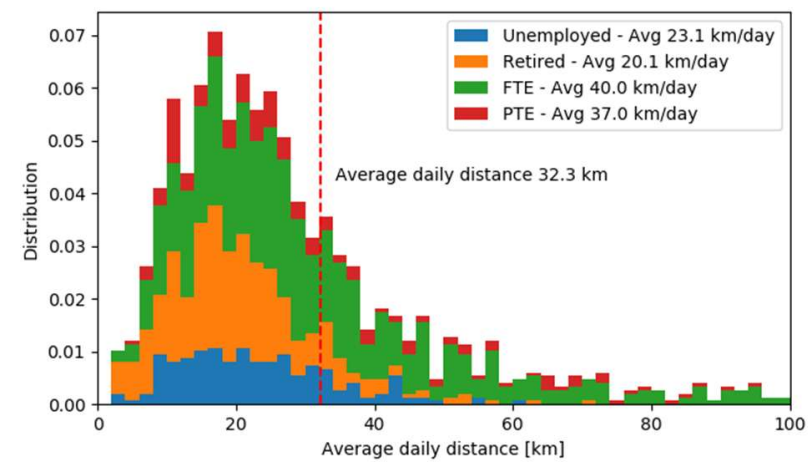

Figure 3: Average daily distance $[\mathrm{km}]$ for different occupant classes (stacked histogram).

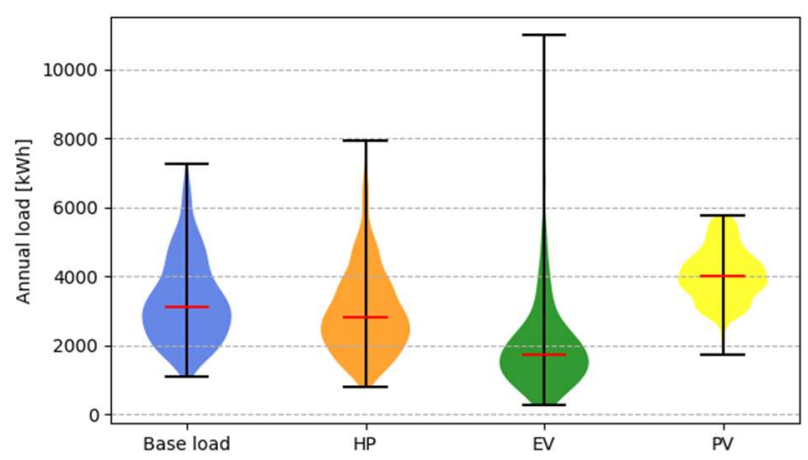

Figure 4: Distribution of annual demand or generation per technology [kWh]. Red bar indicates median value.

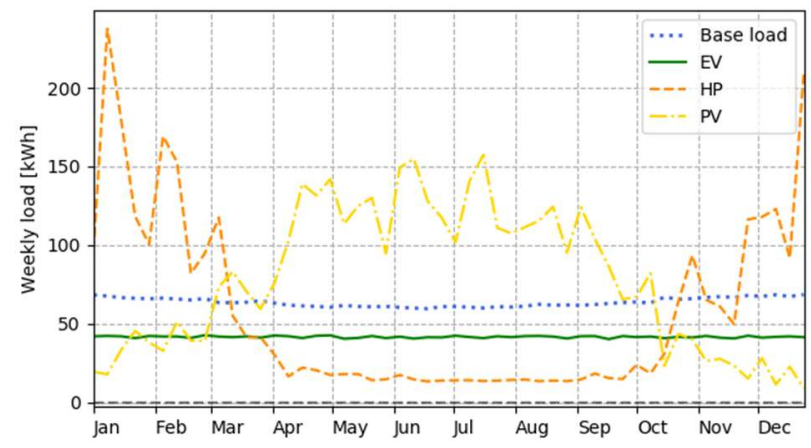

Figure 5: Average weekly demand or generation per technology, per dwelling [kWh]

operation of each technology, as shown in Figure 5 (HP mainly used in winter, PV generation greater in summer and during daytime, EV, and baseload relatively stable along the year), their grid impact will not be the same. This also limits complementarities that may arise between HP and PV.

\section{Results: impact of DER integration and EV smart charging on grid stability}

We realized 20 iterations, each representing a one-year power flow simulation with a different set of dwellings and occupancy profiles, and for each combination of DER penetration integration rate and EV charging strategy. In Figure 6, an example of a dwelling's load profile having the three DERs is shown for both charging strategies. It can be seen that with uncontrolled EV charging, the total household demand can reach over $15 \mathrm{kVA}$ due to the coincidence of HP operation and EV charging. To avoid fuse trips, the user would need to increase the contracted power. The results in this section consider that the user has increased its single-phase contracted power. The smart charging strategy allows to partially avoid this issue. Since there are still few cases where the base load and HP generate peaks greater than the contracted power, controlling the HP demand would be required to never exceed the contracted power. Figure 6 shows as well that users do not charge their EV every day (a non-systematic charging is considered, see section 2.1), which can lead to charging sessions lasting several hours (see Wednesday on bottom plot, Figure 6). 

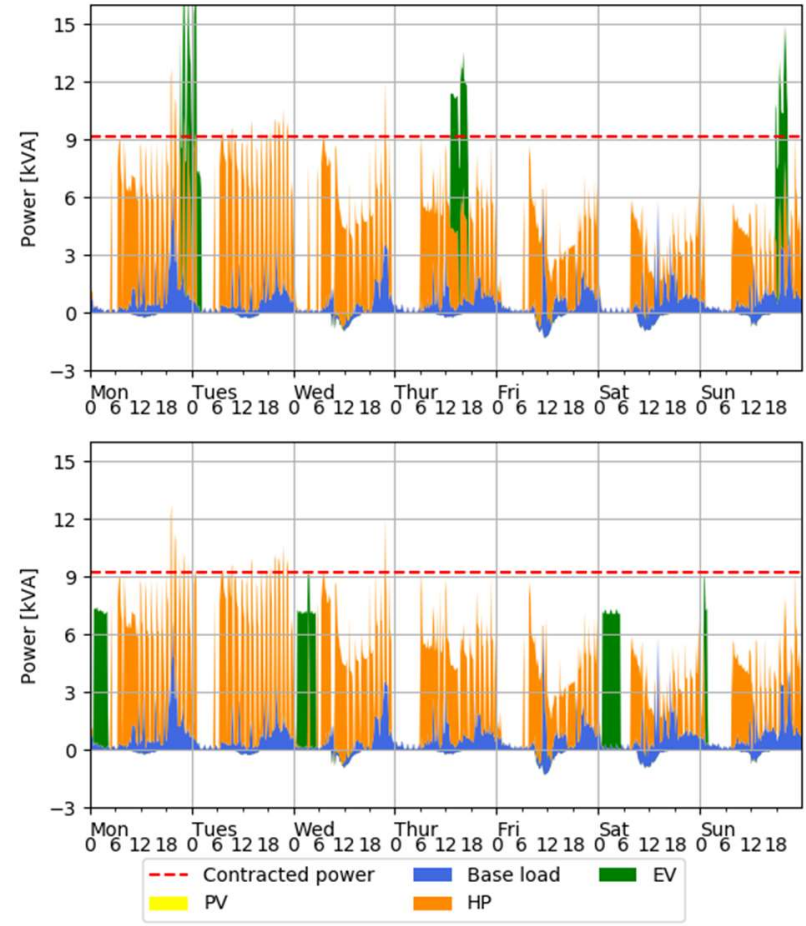

Figure 6: Electricity demand for a dwelling in a winter week. Uncontrolled (top) and smart (bottom) charging.

In Figure 7, we plot the variation of the four grid stability indicators as a function of the DER integration rates and EV charging strategy. Results show that HP have a significantly larger impact than PV and EV on grid stability. Full HP integration creates overloading in the LV transformer of more than $100 \%$ of the transformer rated power, and
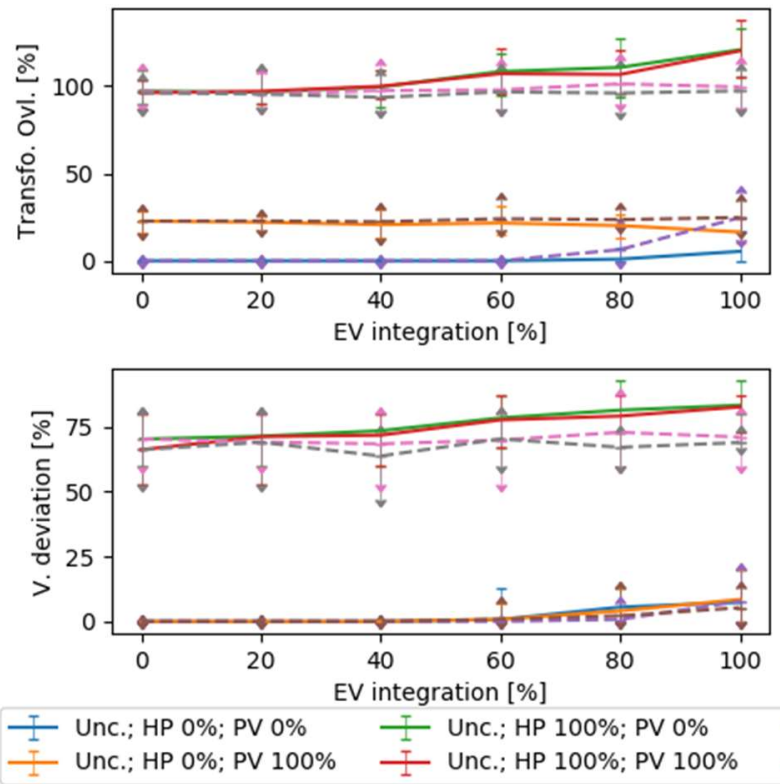

violates voltage deviation and unbalance constraints for $60 \%$ and $20 \%$ of the dwellings respectively. Full PV integration effects are less significant, producing only transformer overloading of $25 \%$ of the transformer rated power. There is no feeder overloading in any $0 \% \mathrm{EV}$ case.

EV integration effects are less significant than full HP integration. Under uncontrolled charging, EVs slightly increase HP-driven transformer overloading and voltage deviations, but have a larger impact on voltage unbalances and create overloading in some phases of the feeder segments. This higher impact on voltage unbalances and feeder overloading can be due to the coincidence of EV charging and HP load on dwellings' single-phase connections, as seen in Figure 6, which create peaks of power demand of $15 \mathrm{~kW}$ on a single phase.

For $0 \% \mathrm{HP}$ cases, uncontrolled EV integration creates grid instabilities only for integration levels above $60 \%$, with voltage deviations for up to $20 \%$ of households and up to $25 \%$ transformer overloading, and no voltage unbalances or overloaded feeder sections.

We observe as well that the smart charging strategy allows to mitigate the impacts of EV integration. For the HP-100\% cases, there is no increase in transformer overloading and voltage deviation indicators, even for high EV penetration cases. Voltage unbalances increase with higher EV penetration, but are less important than in the uncontrolled case, as the single-phase demand of dwellings is limited. No overloaded feeder sections are observed either.

For the HP- $0 \%$ cases, only for EV penetrations above $60 \%$, we observe increasing grid constraints under the smart
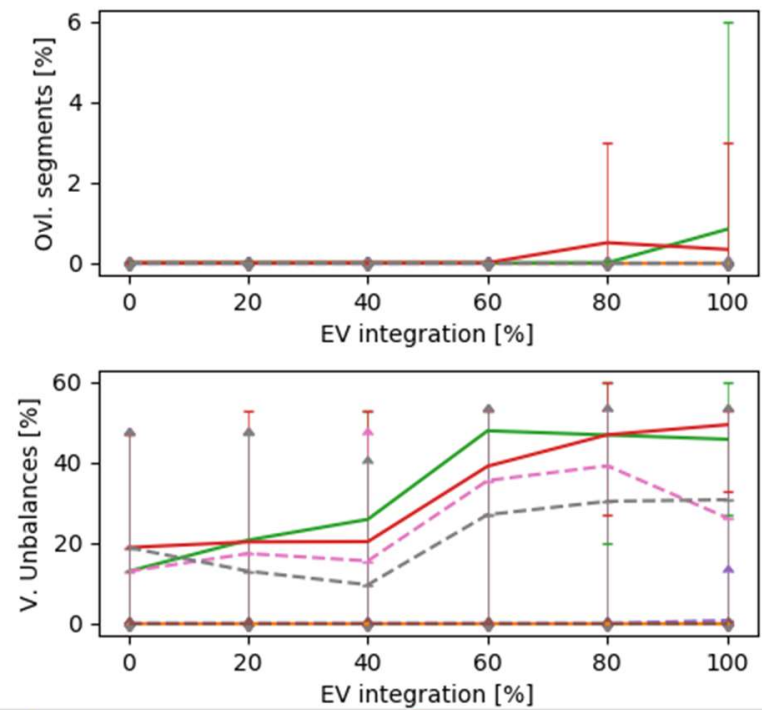

-I- Smart; HP 0\%; PV 0\% - -I- Smart; HP 100\%; PV 0\%

-I- Smart; HP 0\%; PV 100\% -I- Smart; HP 100\%; PV 100\%

Figure 7: Grid stability indicators for different combinations of DER penetration and EV charging strategies.

Transformer overloading (top left), overloaded feeder segments (top right), nodes with voltage deviations (bottom left), and nodes with voltage unbalances (bottom right). Average, minimum, and maximum values for all 20 iterations. 
charge strategy on transformer overloading and voltage deviations. Only in the EV-100\% case, the smart charge strategy underperforms the uncontrolled one, creating higher transformer overloading. This is due to a synchronization of EV load at off-peak hours, whereas with uncontrolled charging, EV load is distributed more evenly along the day. The proposed smart charging does not help reduce the impact of PV integration, as it schedules EV charging at night-time.

\section{Conclusion}

We presented a methodology to evaluate the stability of residential LV grids under large penetration of different distributed energy resources. The methodology is based on an advanced dwelling model that generates detailed load curves for base loads, PV systems, HPs, and EVs from a central occupancy model. Through unbalanced power flow simulations, we identify grids constraints under high DER penetration scenarios. We also evaluate the potential of a decentralized smart charging strategy, which schedules EV charging at night-time off-peak periods while also respecting the dwelling contracted power, to reduce the identified grid instabilities.

We applied the methodology to a typical Belgian LV rural grid. We found that main grid disturbances will come from high HP integration levels, which creates significant transformer overloading and voltage deviation for a high share of dwellings. In contrast, PV creates only transformer overloading and, to a lesser extent, during high-generation and low-demand periods. EV integration impacts are less pronounced than HPs and can create grid instability issues (transformer overloading and voltage deviations) only at high penetration levels (above 60\%).

The proposed smart charging strategy can mitigate EV integration impacts, both for end-users and the grid. For end-users, it allows maintaining the contracted power, thus avoiding connection reinforcements or increases in the energy bill. For the distribution grid, it reduces the grid instability indicators with respect to an uncontrolled charging case, but does not improve them from a non-EV case (i.e. there is no grid benefit from $\mathrm{EV}$ integration, even with smart charging). To effectively reduce grid constraints from PV and HP integration, EV should actively act to reduce grid instability by coordinating charging with PV production or by using bidirectional flows to support the grid during stressed periods created by HPs. These strategies remain for further study.

Given the large impact of HPs on the transformer load (over $2 \mathrm{pu}$ of maximum load), flexibility requirements could be unattainable for EVs alone. A cost-benefit analysis considering the use of flexibility resources (such as V2G and additional stationary batteries) and alternative grid reinforcement should be carried out.

\section{Acknowledgements}

This research benefits from the support of the Chair "Hybrid Technologies and Economy of Electromobility", so-called Armand Peugeot Chair, led by CentraleSupélec and the ESSEC and sponsored by Stellantis, and the financial support of the ANRT for the CIFRE contract $n^{\circ} 2018 / 0031$.

This work is part of the H2020 "sEEnergies" project (https://www.seenergies.eu/). This project has received funding from the European Union's Horizon 2020 Research and Innovation Action under Grant Agreement $N^{\circ} 846463$.

\section{References}

[1] Protopapadaki, C., Saelens, D.: 'Heat pump and PV impact on residential low-voltage distribution grids as a function of building and district properties' Applied Energy, 2017, 192, pp. 268-281.

[2] McKenna, R., Djapic, P., Weinand, et al.: 'Assessing the implications of socioeconomic diversity for low carbon technology uptake in electrical distribution networks', Applied Energy, 2018, 210, pp. 856-869.

[3] Moorman, S., Van 't Wel, T., Van Beek, T.: 'The value of vehicle-to-grid (V2G) for distribution system congestion management', Proc. EVS32 Lyon, May 2019. [4] Navarro-Espinosa, A., Ochoa, L. F.: 'Probabilistic Impact Assessment of Low Carbon Technologies in LV Distribution Systems'. IEEE Transactions on Power Systems, 2016, 31, pp. 2192-2203.

[5] Avramidis, I. I., Evangelopoulos, V. A., Georgilakis, P. S., Hatziargyriou, N. D.: 'Demand side flexibility schemes for facilitating the high penetration of residential distributed energy resources', IET Generation, Transmission \& Distribution, 2018, 12, pp. 4079-4088. [6] Van Roy, J.: 'Electric vehicle charging integration in buildings. Local charging coordination and DC grids', $\mathrm{PhD}$ Thesis, KU Leuven, 2015.

[7] Gonzalez Venegas, F. Petit, M., Perez, Y.: 'Quantifying the participation of EV fleets in local flexibility tenders', Proc. CIRED Berlin Workshop, On-line, 2020.

[8] Meunier, S., Protopapadaki, C., Baetens, R., Saelens, D.: 'Impact of residential low-carbon technologies on lowvoltage grid reinforcements', under review Applied Energy, 2021.

[9] Dixon, J., Bell, K.:'Electric vehicles: Battery capacity, charger power, access to charging and the impacts on distribution networks', eTransportation, 2020, 4, p. 100059.

[10] Western Power Distribution, 'Electric Nation Customer Trial Final Report', 2019. 